\title{
Téoros
}

Revue de recherche en tourisme

\section{Portrait du tourisme à Madagascar} Entre nature et éloignement

\section{Jean Stafford, Bruno Sarrasin et Haja Ramahatra}

Volume 21, numéro 2, été 2002

URI : https://id.erudit.org/iderudit/1072410ar

DOI : https://doi.org/10.7202/1072410ar

Aller au sommaire du numéro

Éditeur(s)

Université du Québec à Montréal

ISSN

0712-8657 (imprimé)

1923-2705 (numérique)

Découvrir la revue

Citer cette note

Stafford, J., Sarrasin, B. \& Ramahatra, H. (2002). Portrait du tourisme à

Madagascar : entre nature et éloignement. Téoros, 21(2), 70-72.

https://doi.org/10.7202/1072410ar d'utilisation que vous pouvez consulter en ligne.

https://apropos.erudit.org/fr/usagers/politique-dutilisation/ 


\section{Portrait du tourisme à Madagascar}

Entre nature et éloignement

\section{Jean Stafford, Bruno Sarrasin et Haja Ramahatra}

Cette chronique porte sur la conjoncture touristique. Au cours des cinq dernières années, nous y avons présenté un certain nombre de clientèles touristiques du Québec (Étatsuniens, Français, Allemands, Britanniques, etc.), un ensemble d'enjeux pour l'industrie (saisonnalité, emplois, offre hôtelière, etc.) et quelques exemples internationaux (Maroc et Viêt-Nam). Dans ce numéro nous proposons un portrait simplifié de la situation du tourisme à Madagascar à partir des données disponibles. Les notions usuelles de l'analyse prévisionnelle seront utilisées, telles la tendance (passée, présente et future) et la saisonnalité et des prévisions allant jusqu'en 2005 seront présentées.

\section{Portrait du tourisme à Madagascar}

D epuis quelques années, le marché du tourisme dans la région de 'océan indien progresse à un rythme annuel moyen de 9 à $13 \%$, proche du double du rythme moyen mondial $(5,5 \%)^{1}$. Les grandes destinations comme la Réunion, les Seychelles et l'île Maurice sont davantage visitées et cette dernière se taille la part du lion en captant plus de la moitié du trafic aérien régional. L'île de Madagascar, quant à elle, représente une destination encore peu explorée et réalise une modeste performance, aussi bien sur le marché mondial $(0,01 \%$ de part de marché) que sur le marché océano-indien².
À Madagascar, le nombre de voyageurs non-résidents a crû en moyenne de 11,7\% par année ${ }^{3}$, de 1990 à 2000 (tableau 1). Malgré cela, le nombre d'arrivées en 2000 demeurait très modeste sur la Grande île avec 160071 touristes, alors que ses atouts - principalement naturels - lui conféreraient une demande possible de 250000 à 450000 touristes par $\mathrm{an}^{4}$.

Le tourisme occupe une place de première importance à Madagascar. Sa contribution économique, plus spécifiquement mesurée en apport de devises, dépasse celle des autres grandes industries d'exportation comme la crevette, le café ou la vanille (tableau 2). Bien que relativement modeste sur le plan des arrivées, les tableaux 1 et 2 montrent que l'industrie du tourisme est en croissance depuis 1992. Cependant, les événements politiques survenus depuis janvier 2002 auront certainement des effets néfastes sur l'industrie touristique malgache à cause des restrictions politiques appliquées aux transporteurs aériens, des blocus des provinces, de la dégradation de la sécurité, etc. Ces conditions ressemblent à celles qui ont prévalu à Madagascar en 1991, entraînant une chute de $34 \%$ des arrivées de voyageurs non-résidents en comparaison à 1990.

\section{L'offre hôtelière et les produits touristiques}

Le nombre d'infrastructures hôtelières malgaches a peu évolué ces dernières années. La capacité totale des offres en 2000 a été évaluée à 7779 chambres (contre 6066 en 1996) réparties dans 644 établissements hôteliers de différentes catégories (contre 424 en

TABIEAU 1

Évolution du nombre de voyageurs non-résidents (1990 à 2000) et des recettes touristiques (1992 à 2000) à Madagascar

\begin{tabular}{|c|c|c|c|c|}
\hline Année & $\begin{array}{l}\text { Nombre de voyageurs } \\
\text { non-résidents } \\
\text { de } 1990 \text { à } 2000^{\circ}\end{array}$ & $\begin{array}{c}\text { Evolution indicielle de } \\
1990 \text { à } 2000(100=1990)\end{array}$ & $\begin{array}{l}\text { Recettes touristiques en } \\
\text { millions de francs malgaches } \\
\text { (FMG) de } 1992 \text { à } 2000^{\mathrm{b}}\end{array}$ & $\begin{array}{l}\text { Recettes touristiques } \\
\text { en millions de SCAN } \\
\text { de } 1992 \text { à } 2000^{\circ}\end{array}$ \\
\hline 1990 & 52923 & 100 & n.d. & n.d. \\
\hline 1991 & 34891 & 66 & n.d. & n.d. \\
\hline 1992 & 53656 & 101 & 69250 & 16,18 \\
\hline 1993 & 65102 & 121 & 75856 & 17,73 \\
\hline 1994 & 65839 & 124 & 141657 & 33,11 \\
\hline 1995 & 74619 & 141 & 171760 & 40,14 \\
\hline 1996 & 82681 & 156 & 262925 & 61,45 \\
\hline 1997 & 100762 & 190 & 371146 & 86,74 \\
\hline 1998 & 121207 & 229 & 483586 & 113,01 \\
\hline 1999 & 138253 & 261 & 624988 & 146,06 \\
\hline 2000 & 160071 & 302 & 821034 & 191,88 \\
\hline
\end{tabular}

- Ministère du Tourisme - 2001

Banque centrale de la République de Madagascar - 2001

$1 \$ C A N=4279$ FMG au $13 / 09 / 02$ 
1996). Par rapport à l'année 1996, l'augmentation de l'offre en chambres a été de $28 \%{ }^{5}$.

Face à la prolifération des établissements irréguliers et hors normes, la direction de Suivi et de Contrôle du ministère du Tourisme a procédé à l'examen continu des établissements et à leur classement ${ }^{6}$. Ainsi, depuis l'année 2000, 67 établissements ont fait l'objet d'une réévaluation et d'un classement répondant réellement aux normes requises par les voyagistes internationaux. Bien que l'on puisse reconnaitre l'effort entrepris par le ministère du Tourisme pour rehausser la qualité de l'hébergement sur la Grande île, l'offre d'établissements hôteliers demeure très inégale et mal répartie géographiquement?

- $37 \%$ du parc hôtelier et $51 \%$ des chambres de 3 à 5 étoiles se concentrent à Antananarivo, la capitale ;

- les villes des régions centrales et les hautes terres (Antsirabe, Fianarantsoa, Ambatolampy, Moramanga, Ranomafana) représentent $28 \%$ de l'offre hôtelière ;

- les sites balnéaires (Nosy Be, Fort Dauphin, Sainte Marie, Toliara, Morondava, Diégo, Majunga, Tamatave, Antalaha, Sambava, Manakara, Mananjary) représentent $35 \%$ des chambres disponibles ;

- avec plus de $18 \%$ des chambres, Nosy Be est le principal pôle de concentration touristique balnéaire.

Bien que l'image de Madagascar évolue de plus en plus vers l'écotourisme, l'essentiel de la demande est de type balnéaire et se développe autour de quelques villes secondaires de la côte accessibles de la capitale par avion. Le profil socio-démographique des visiteurs est assez typé : il s'agit d'une population dont l'âge se situe de 25 à 30 ans, constituée majoritairement de diplômés. L'origine sociale élevée des visiteurs est confirmée par leur appartenance à une catégorie socioprofessionnelle élevée (cadre - profession libérale). La plupart des touristes viennent durant la période allant de juillet à septembre, à l'exception des Allemands qui viennent surtout durant les mois de décembre, janvier, février et mars et qui dépensent en moyenne 3200 \$CAN pour une durée moyenne de 20 jours. Les gens d'affaires dépensent beaucoup plus (3800\$CAN) que les personnes venues visiter leurs proches (2 656 \$CAN). Ajoutons que le budget est aussi fonction du type de voyage et de la durée du séjour ${ }^{8}$.

\section{L'écotourisme}

L'écotourisme connaît la croissance la plus rapide de toute l'industrie du voyage à Madagascar avec un taux annuel de 10 à $30 \%$ contre une croissance globale du tourisme de $4 \%$ par an ${ }^{9}$. Sur le marché touristique malgache, on note en particulier une hausse de la demande de produits écotouristiques axés sur la visite d'aires protégées. Les statistiques d'entrées compilées par l'ANGAP (Association nationale pour la gestion des aires protégées) depuis 1992 traduisent un intérêt de plus en plus marqué pour les parcs et les réserves naturelles de l'île (tableau 3).

En s'appuyant sur les quelques données touristiques disponibles à Madagascar, il est possible d'évaluer de façon approximative la proportion des écotouristes en comparant le nombre total des visiteurs étrangers qui pénètrent dans les aires protégées par rapport au total des arrivées touristiques dans une même année. Selon ces calculs, les écotouristes constituent $43 \%$ de la demande touristique totale de Madagascar ${ }^{10}$.

Comme le présente le tableau 4, la grande majorité des arrivées de touristes étrangers à Madagascar provient de l'Europe (75\% des écotouristes et $82 \%$ des touristes). Par ailleurs, la proportion d'écotouristes $(43 \%)$ par rapport à la demande touristique totale est appelée à croître, compte tenu du potentiel de Madagascar (diversité des paysages, faune et flore, richesse éthno-culturelle, diversité de ses sites naturels : grottes, formations calcaires, aires protégées, etc.).

\section{Quelques perspectives d'avenir}

Sur le plan touristique, Madagascar possède un certain nombre d'atouts qui représentent autant d'avantages comparatifs face aux autres destinations de l'océan Indien" ${ }^{1 !}$

- Sa géographie diversifiée autorise un tourisme aussi bien sédentaire (séjour balnéaire) qu'itinérant (circuits de découverte et d'ouverture) et mixte (circuits combinés à un séjour), ainsi que marginalement un tourisme de croisière et de plaisance ;

- Son image, bien que fragile et à construire, permet d'envisager un tourisme à budget élevé aussi bien qu'à budget moyen ;

- Son potentiel socioéconomique peut générer un tourisme captif non négligeable, qu'il relève du tourisme d'affaires ou du tourisme d'affinité.

\begin{tabular}{|c|c|c|c|c|c|c|c|c|c|}
\hline & & & touristiqu & principaux p & $\begin{array}{l}\text { U } 2 \\
\text { dexporti }\end{array}$ & $97-2000)$ & CAN & & \\
\hline & oduits & & & & & & & & \\
\hline & & milliards & millions & milliards & millions & milliards & millions & milliards & millions \\
\hline & & de FMG & de \$CAN & de FMG & de $\$ C A N$ & de FMG & de SCAN & de FMG & de SCAN \\
\hline $1^{\text {er }}$ & Tourisme & 371,146 & 86,736 & 483,586 & 113,013 & 624,988 & 146,059 & 821,034 & 191,875 \\
\hline $2^{\circ}$ & Crevette & 328,348 & 76,734 & 375,794 & 87,823 & 496,212 & 115,964 & 625,380 & 146,150 \\
\hline $3^{e}$ & Café & 168,383 & 39,351 & 217,060 & 50,726 & 188,011 & 43,938 & 55,390 & 12,944 \\
\hline $4^{\circ}$ & Vanille & 48,761 & 11,395 & 129,909 & 30,359 & 173,417 & 40,527 & 138,476 & 32,362 \\
\hline & Total & 2573,468 & 601,418 & 2824,735 & 660,139 & 3656,351 & 854,487 & 5564,988 & 1300,534 \\
\hline
\end{tabular}

Source : Banque centrale de la République de Madagascar - 2001. 


\begin{tabular}{|c|c|c|}
\hline \multicolumn{3}{|c|}{ TABLEAU 3 } \\
Évolution des entrées dans les réserves de YANGAP, de $\mathbf{1 9 9 2}$ à $\mathbf{2 0 0 0}$ \\
\hline Année & Nombre d'entrées & Indice $100=1992$ \\
\hline 1992 & 5898 & 100 \\
\hline 1993 & 14962 & 254 \\
\hline 1994 & 17418 & 295 \\
\hline 1995 & 36236 & 614 \\
\hline 1996 & 41934 & 711 \\
\hline 1997 & 50333 & 853 \\
\hline 1998 & 69981 & 1187 \\
\hline 1999 & 79812 & 1353 \\
\hline 2000 & 84000 & 1424 \\
\hline
\end{tabular}

Source: ANGAP - 2001

Ces conditions ont amené le gouvernement malgache à se fixer comme objectif l'atteinte de 400000 touristes en $2005^{12}$. Cet objectif est conditionnel à :

- une augmentation de la capacité du transport aérien ;

- une augmentation des infrastructures de liaison et d'équipements sociaux collectifs ;

- une amélioration de la compétence des opérateurs par une information, une formation et l'expérience suffisantes ;

- une amélioration de l'organisation et de la gestion de l'activité touristique en renforçant l'image touristique malgache pour une meilleure compétitivité ;

- une diversification et une concentration des activités dans des espaces sélectionnés.

Les objectifs énoncés par le gouvernement malgache et les actions à entreprendre pour y arriver nous semblent irréalistes. L'évolution observée de 1990 à 2000 ne permet cependant pas de soutenir un tel scénario de croissance des arrivées. Comme le montre le tableau 5 , les deux modèles prévisionnels utilisés ${ }^{13}$ présentent des arrivées de voyageurs non-résidents de 175000 à 230000 à l'horizon 2003 et, si nous prenons le risque de pousser nos prévisions davantage ${ }^{14}$, seul l'intervalle supérieur de la tendance exponentielle permet d'atteindre l'objectif que le gouvernement s'est fixé. Ces conditions, bien qu'elles soient à la limite du possible sur le plan statistique, ne tiennent pas compte des récents problèmes socio-politiques qui se sont déroulés au cours de l'année 2002 et qui auront très cer-

\begin{tabular}{|l|c|c|}
\hline \multicolumn{3}{|c|}{$\begin{array}{c}\text { TABIEAU 4 } \\
\text { Comparaison entre touristes et écotouristes, selon le pays de résidence (en 2000) }\end{array}$} \\
\hline $\begin{array}{l}\text { Pays de } \\
\text { résidence }\end{array}$ & $\begin{array}{c}\text { Touristes } \\
\text { (\% par rapport au total } \\
\text { des arrivées touristiques) }\end{array}$ & Écotouristes \\
\hline France & $30,5 \%$ & $32 \%$ \\
\hline Réunion & $6 \%$ & $4,5 \%$ \\
\hline ltadlie & $8,8 \%$ & $10 \%$ \\
\hline Angleterre & $6 \%$ & $6,5 \%$ \\
\hline Allemagne & $20 \%$ & $7 \%$ \\
\hline Autres pays d'Europe & $11 \%$ & $15 \%$ \\
\hline États-Unis et Canada & $6,8 \%$ & $9,2 \%$ \\
\hline Afrique du Sud & $0,8 \%$ & $4,4 \%$ \\
\hline Maurice & $1,5 \%$ & $0,1 \%$ \\
\hline Autres & $7,5 \%$ & $11,3 \%$ \\
\hline Source : Enquête du bureau d'études ORGASYS / Ministère du Tourisme er ANGAP - 2000 \\
\hline
\end{tabular}

\begin{tabular}{|l|c|c|}
\hline & TABLEAU 5 \\
& $\begin{array}{c}\text { Prévisions des arrivées de voyageurs non-résidents, } \\
\text { selon deux modéles mathématiques, de } \mathbf{2 0 0 1} \text { à } \mathbf{2 0 0 5}\end{array}$ \\
\hline Année & Tendance linéaire & Tendance exponentielle \\
\hline 2001 & 154003 & 174197 \\
\hline 2002 & 165276 & 198899 \\
\hline 2003 & 176549 & 227105 \\
\hline 2004 & 187822 & 259310 \\
\hline 2005 & 199094 & 296082 \\
\hline
\end{tabular}

tainement un effet sur la perception de la Grande île bien au-delà de cette année. Bien que Madagascar possède un potentiel touristique en grande partie sous-exploité, nous croyons que l'évolution des arrivées de voyageurs non-résidents sur la Grande île prendra une forme davantage linéaire - avec une chute en 2001 - qu'exponentielle. Cette croissance modérée peut représenter une réelle opportunité pour l'industrie touristique malgache de mieux structurer et diversifier son offre de façon à répondre non seulement à la demande internationale, mais aussi à la demande intérieure en émergence.

Jean Stafford est professeur au département d'études urbaines et touristiques de l'UQAM, Bruno Sarrasin est professeur substitut au département d'études urbaines et touristiques de l'UQAM et Haja Ramahatra est étudiant au MBA Recherche à l'UQAM.

\section{Notes}

1 Évaluations du ministère du Tourisme de la République de Madagascar, 2000.

2 Idem.

3 Moyenne géométrique.

4 Voir le « Projet de développement du tourisme à Madagascar : Études d'impacts du tourisme », ministère du Tourisme / Programme des nations unies pour le développement (PNUD) - 1992.

5 Ministère du Tourisme - 2001

6 Voir notamment la loi n ${ }^{\circ} 95-017$ du 25 août 1995 , le décret n ${ }^{\circ} 2001$ 027 portant refonte du décret 96773 du 3 septembre 1996 et les arrêtés du 19 avril 2001 qui fixent les normes exigées pour chaque catégorie d'établissements.

7 Ministère du Tourisme - 2001.

8 Ministère du Tourisme (2001), Enquête tourisme 2000, Projet MADIO, République de Madagascar, Antananarivo.

9 Ministère du Tourisme / ANGAP - 2001.

$10 \mathrm{Idem}$.

11 Voir à ce sujet, Programme des nations unies pour le développement (PNUD) et ministère du Tourisme (2000), Perspective touristique pour Madagascar, République de Madagascar, Antananarivo.

12 Ministère du Tourisme - 2001.

$13 \mathrm{R}^{2} \geq 0,9$, tests de Fisher et Student significatifs au seuil de $5 \%$.

14 Pour faire des prévisions jusqu'en 2005, il aurait fallu idéalement avoir 15 années d'observations, soit de 1986 à 2000 ou de 1987 à 2001. 